\title{
Temporal stability of multitrigger and episodic viral wheeze in early childhood
}

\author{
Ben D. Spycher ${ }^{1,2}$, Cara Cochrane ${ }^{3}$, Raquel Granell ${ }^{1}$, Jonathan A.C. Sterne ${ }^{1}$, \\ Michael Silverman ${ }^{4}$, Eva Pedersen ${ }^{2}$, Erol A. Gaillard ${ }^{5,6,7}$, John Henderson ${ }^{1}$ and \\ Claudia E. Kuehni ${ }^{2,8}$
}

Affiliations: ${ }^{1}$ Population Health Sciences, Bristol Medical School, University of Bristol, Bristol, UK. ${ }^{2}$ Institute of Social and Preventive Medicine (ISPM), University of Bern, Bern, Switzerland. ${ }^{3}$ Paediatric Respiratory Dept, Bristol Royal Hospital for Children, Bristol, UK. ${ }^{4}$ University of Leicester, Leicester, UK. ${ }^{5}$ Institute for Lung Health, NIHR Leicester Respiratory Biomedical Research Unit, University of Leicester, Leicester, UK. ${ }^{6}$ Dept of Infection Immunity and Inflammation, University of Leicester, Leicester, UK. ${ }^{7}$ University Hospitals Leicester, Children's Hospital, Leicester, UK. ${ }^{8}$ Paediatric Respiratory Medicine, Children's University Hospital of Bern, University of Bern, Bern, Switzerland.

Correspondence: Ben D. Spycher, Institute of Social and Preventive Medicine (ISPM), University of Bern, Finkenhubelweg 11, CH-3012 Bern, Switzerland. E-mail: ben.spycherdispm.unibe.ch

@ERSpublications

Multitrigger and episodic viral wheeze tend to persist in early childhood and may reflect distinct disease processes http://ow.ly/KQrk30fvXMj

Cite this article as: Spycher BD, Cochrane C, Granell R, et al. Temporal stability of multitrigger and episodic viral wheeze in early childhood. Eur Respir J 2017; 50: 1700014 [https://doi.org/10.1183/ 13993003.00014-2017].

ABSTRACT The distinction between episodic viral wheeze (EVW) and multitrigger wheeze (MTW) is used to guide management of preschool wheeze. It has been questioned whether these phenotypes are stable over time. We examined the temporal stability of MTW and EVW in two large population-based cohorts.

We classified children from the Avon Longitudinal Study of Parents and Children $(n=10970)$ and the Leicester Respiratory Cohorts ((LRCs), $\mathrm{n}=3263$ ) into EVW, MTW and no wheeze at ages 2, 4 and 6 years based on parent-reported symptoms. Using multinomial regression, we estimated relative risk ratios for EVW and MTW at follow-up (no wheeze as reference category) with and without adjusting for wheeze severity.

Although large proportions of children with EVW and MTW became asymptomatic, those that continued to wheeze showed a tendency to remain in the same phenotype: among children with MTW at 4 years in the LRCs, the adjusted relative risk ratio was 15.6 (95\% CI 8.3-29.2) for MTW (stable phenotype) compared to 7.0 (95\% CI 2.6-18.9) for EVW (phenotype switching) at 6 years. The tendency to persist was weaker for EVW and from 2-4 years. Results were similar across cohorts.

This suggests that MTW, and to a lesser extent EVW, tend to persist regardless of wheeze severity.

Received: Jan 042017 | Accepted after revision: July 292017

This article has supplementary material available from erj.ersjournals.com

Editorial comment in: Eur Respir J 2017; 50: 1701283.

Copyright OERS 2017 


\section{Introduction}

There is debate whether recurrent wheezing in young children represents a single disease entity, 'childhood asthma', or a heterogeneous group of disorders, referred to as asthma 'phenotypes'. Numerous attempts have been made to distinguish phenotypes [1-3]. A commonly used classification is the distinction between episodic viral wheeze and multitrigger wheeze $[4,5]$. Episodic viral wheeze (EVW), also called exclusive viral wheeze, characterises children who wheeze only during respiratory infections. During the intervals between colds, these children are asymptomatic. EVW is frequent in infancy and preschool years, and less prevalent in older children [6], and has also been described in adults [7]. Multitrigger wheeze (MTW) more closely resembles classical asthma [8]. Children with MTW also wheeze between respiratory infections in response to a variety of factors, including allergens, exercise, laughing or crying, strong smells or certain foods or drinks [9]. MTW is more strongly associated with lung function abnormalities [8] and atopy [10]. While most children with EVW become asymptomatic, MTW tends to persist [11, 12]. This two-phenotype model has been used to inform the management of preschool wheeze [9, 13-16]. For instance, a European Respiratory Society (ERS) taskforce recommended using inhaled corticosteroids for maintenance treatment of MTW, but montelukast for EVW [9].

The distinction between EVW and MTW and its usefulness for the management of preschool wheeze has been challenged [17, 18]. GarCia-Marcos and Martinez [19] suggested that the two phenotypes merely reflect the ends of a severity spectrum, with MTW representing more severe wheeze. Severity of wheeze, in particular frequency of episodes, strongly predicts long-term prognosis [12, 20, 21]. It has also been questioned whether these phenotypes are sufficiently stable over time to represent clinically meaningful entities $[22,23]$. In an update of their 2014 recommendations, the ERS taskforce pointed out that wheeze patterns in young children vary over time and with treatment, rendering the distinction between EVW and MTW difficult in many patients [17]. Consequently, inhaled corticosteroids remained the first-line treatment for MTW, but were also recommended for patients with frequent or severe EVW. The taskforce concluded that future research should focus on disease severity in addition to phenotypes [17].

The current study used longitudinal data on wheezing at ages 2,4 and 6 years from two large population-based birth cohorts, to examine the stability of MTW and EVW over time, and the degree to which stability was explained by differences in wheeze severity.

\section{Materials and methods}

Study populations

ALSPAC (Avon Longitudinal Study of Parents and Children) is a longitudinal population-based birth cohort study that recruited 14541 pregnant women resident in Avon, UK, with expected dates of delivery between April 1991 and December 1992. There were 14062 live-born children. The study has been described in detail elsewhere [24]. Each year up until the children were 8 years of age, the study mothers were sent child health questionnaires including detailed questions on respiratory symptoms. Ethical approval was obtained from the ALSPAC Ethics and Law Committee and from local research ethics committees.

The Leicester 1998-b respiratory cohort (LRC) consists of a population-based random sample of 4300 children born between May 1996 and April 1997 in Leicestershire, UK. It is described in detail elsewhere [25]. Perinatal routine data were obtained from the Leicestershire Health Authority Child Health Database, and mothers were sent questionnaires including detailed questions on respiratory symptoms in 1998, 1999, 2001, 2003, 2006 and 2010. The study was approved by the Leicestershire Health Authority Research Ethics Committee.

Support statement: This study was supported by the Swiss National Foundation (Grant nos. SNF32003B_162820 and SNF32003B_144068). The UK Medical Research Council and the Wellcome Trust (grant no. 092731) and the University of Bristol provided core support for the ALSPAC (Avon Longitudinal Study of Parents and Children) study. In the Leicester Respiratory Cohort study, data collection was funded by the UK National Asthma Campaign, the University Hospitals of Leicester NHS Trust (R\&D), Leicestershire and Rutland Partnership Trust, Medisearch, the Trent NHS Regional Health Authority, and the UK Department of Health. B.D. Spycher is the recipient of a European Respiratory Society (ERS)/Marie Curie Joint Research Fellowship (grant no. MC 1614-2010). The research leading to these results has received funding from the ERS and the European Community's Seventh Framework Programme FP7/ 2007-2013-Marie Curie Actions under grant agreement RESPIRE, PCOFUND-GA-2008-229571. B.D Spycher also received support from a Swiss National Science Foundation fellowship (grant no. PZ00P3_147987). R. Granell was supported by the UK Medical Research Council (grant no. G0902125). J.A.C. Sterne is funded by the National Institute for Health Research Senior Investigator award NF-SI-0611-10168. The funders had no role in the study design, data collection and analysis, the decision to publish or the preparation of the manuscript. Funding information for this article has been deposited with the Crossref Funder Registry.

Conflict of interest: Disclosures can be found alongside this article at erj.ersjournals.com 
We included all children in both cohorts whose parents responded to a questionnaire sent at age 2, 4 or 6 years (or a questionnaire sent at age 30, 57 and 81 months for ALSPAC).

\section{Definition of wheeze phenotypes}

The questions used to address wheeze or whistling in the previous 12 months ('current wheeze') were similar in both cohorts (table 1). Children were assigned to the EVW phenotype if they reported current wheeze in the previous 12 months with infections as a trigger and no other triggers (table 1). Children with current wheeze in the previous 12 months reporting a trigger category other than infections were assigned to MTW. Children with current wheeze who could not be assigned either to EVW or MTW were designated non-classifiable.

\section{Information on wheeze severity}

We defined the following indicators of wheeze severity based on symptoms in the previous 12 months: frequent wheeze attacks ( $\geqslant 3$ in ALSPAC, $\geqslant 4$ in LRC), shortness of breath during wheeze attacks, sleep disturbed due to wheezing, speech limited to one to two words at a time between breaths due to wheeze (ALSPAC only), and wheeze interfering with the child's daily activities (LRC only). The questions used to assess this information and the definitions of severity indicators are provided in supplementary table S1.

\section{Statistical analysis}

We carried out the following analysis steps:

1) We computed the prevalence of current wheeze, EVW and MTW at ages 2, 4 and 6 years.

2) At each age, we assessed the association between wheeze phenotypes and dichotomous indicators of severity (supplementary table S1) by calculating the odds ratios for MTW versus EVW, comparing severe with less severe wheeze using logistic regression.

3) For each age interval (2-4, 4-6 and 2-6 years), we assessed whether wheeze phenotype at the first time point (baseline) predicted current wheeze at the later time point (follow-up). We used logistic regression

TABLE 1 Questionnaire items and definitions of wheeze phenotypes in the Avon Longitudinal Study of Parents and Children (ALSPAC) and the Leicester Respiratory Cohort Study (LRC)

\section{ALSPAC}

\section{LRC}

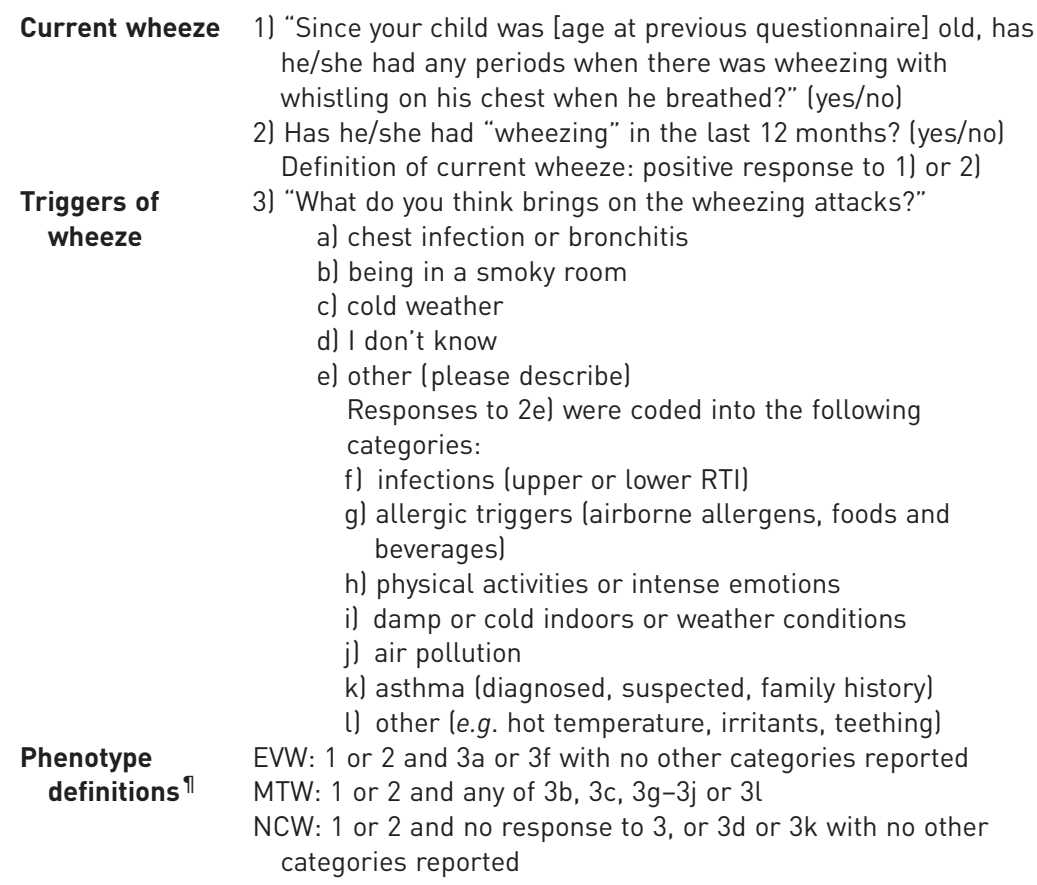

Current wheeze 1) "Since your child was [age at previous questionnaire] old, has he/she had any periods when there was wheezing with whistling on his chest when he breathed?" (yes/no)

2) Has he/she had "wheezing" in the last 12 months? (yes/no) Definition of current wheeze: positive response to 1) or 2]

Triggers of wheeze

3) "What do you think brings on the wheezing attacks?"

a) chest infection or bronchitis

b) being in a smoky room

c) cold weather

d) I don't know

e) other (please describe)

Responses to 2e) were coded into the following categories:

f) infections (upper or lower RTI)

g) allergic triggers lairborne allergens, foods and beverages)

h) physical activities or intense emotions

i) damp or cold indoors or weather conditions

j) air pollution

k) asthma (diagnosed, suspected, family history)

l) other (e.g. hot temperature, irritants, teething)

Phenotype

definitions $^{\text {ๆ }}$

EVW: 1 or 2 and $3 a$ or $3 f$ with no other categories reported

MTW: 1 or 2 and any of $3 b, 3 c, 3 g-3 j$ or $3 l$

NCW: 1 or 2 and no response to 3 , or $3 d$ or $3 k$ with no other categories reported

1) "Has your child had wheezing or whistling in the chest in the last 12 months?" (yes/no) Definition of current wheeze: positive response to 1)

2) "In the last 12 months, has your child had wheezing or whistling in the chest during or soon after a cold or flu?" (yes/no)

3) "In the last 12 months, has your child had wheezing or whistling in the chest even without having a cold or flu?" (yes/no)

4) "In the last 12 months, did the following things cause wheezing in your child?"

a) exercise (playing or running)

b) laughing, crying or excitement

c) contact with pets or other animals

d) pollen (grass, hay, trees, flowers)

e) food or drinks

(answer categories for a-d: yes/no/don't know)

EVW: 1 and 2 with no positive response to any of $3,4 a-$

$4 \mathrm{e}$

MTW: 1 and any of $3,4 a-4 e$

NCW: 1 and no positive response to any of 2, 3, 4a-4e

RTI: respiratory tract infection; EVW: episodic viral wheeze; MTW: multitrigger wheeze; NCW: non-classifiable wheeze." : only asked from age 4 years onwards; ${ }^{\text {? }}$ : positive responses to listed questionnaire items required. 
to estimate the odds ratios for current wheeze at follow-up, comparing children with EVW and MTW at baseline with those without wheeze.

4) For each age interval, we assessed whether children tended to have the same wheeze phenotypes at follow-up as they did at baseline. We first calculated the probability for these categories at follow-up given the category at baseline. Using multinomial logistic regression, we then estimated the relative risk ratios (RRRs) for EVW and MTW at follow-up, comparing these phenotypes with no wheeze at baseline. We adjusted the regression models for symptom severity (original variables, not dichotomised) at baseline to determine whether the phenotypes at baseline predicted the phenotypes at follow-up independent of severity. In separate models we additionally adjusted for sex, ethnicity (white, other), maternal smoking during pregnancy, older siblings (yes/no), crowding (>1 person/room) and pet ownership. The RRRs compared the risk ratio for phenotypes at follow-up (probability of having the phenotype divided by probability of having no wheeze) in children of a given phenotype at baseline (EVW or MTW) to children with no wheeze at baseline. We also tested for the equality of RRRs between EVW and MTW at baseline. Such equality implies absence of phenotype persistence. For instance, equality of RRRs for EVW at follow-up means that after excluding children with MTW at follow-up, those with EVW and MTW at baseline are equally likely to have EVW at follow-up.

\section{Results}

Of the 14062 live-born children recruited in ALSPAC, we included 10970 (78\%) for whom information on wheeze was available for at least one time point (age 2, 4 or 6 years). Information on wheeze was provided for 9953, 9391 and 8393 children at the ages of 2, 4 and 6 years (table 2). Similarly, of the 4300 children in the LRC (1998-b cohort), we included 3263 (76\%) and information on wheeze was reported for 2355, 2609 and 2077 at ages 2, 4 and 6 years respectively.

The cohorts differed with respect to ethnicity and socioeconomic conditions (table 2). In ALSPAC, 97\% of the children were white. In the LRC, $85 \%$ were white and $15 \%$ of South Asian origin. Households in the LRC tended to be more crowded, and maternal smoking and pet ownership were less common than in ALSPAC. The proportions of children whose mothers smoked during pregnancy, who had older siblings

TABLE 2 Characteristics of the study populations (Avon Longitudinal Study of Parents and Children (ALSPAC) and the Leicester Respiratory Cohort (LRC) study) and prevalence of wheeze phenotypes at ages 2, 4 and 6 years

\begin{tabular}{|c|c|c|c|c|}
\hline \multirow[t]{2}{*}{ Characteristics } & \multicolumn{2}{|c|}{ ALSPAC } & \multicolumn{2}{|c|}{ LRC } \\
\hline & $\mathrm{n} / \mathrm{N}$ & $\%$ & $\mathrm{n} / \mathrm{N}$ & $\%$ \\
\hline \multicolumn{4}{|l|}{ Sociodemographic data } & \\
\hline Male sex & $5680 / 10970$ & 52 & $1692 / 3263$ & 52 \\
\hline Ethnicity white \# $^{\#}$ & $10266 / 10574$ & 97 & $2761 / 3263$ & 85 \\
\hline Maternal smoking in pregnancy & $2635 / 10879$ & 24 & $460 / 2865$ & 16 \\
\hline$\geqslant 1$ older siblings & $5778 / 10274$ & 56 & $1837 / 2798$ & 66 \\
\hline Crowding $>1$ person in room & $2285 / 9406$ & 24 & $1150 / 2852$ & 40 \\
\hline Pet owner & $5475 / 9805$ & 56 & $1226 / 2903$ & 42 \\
\hline \multicolumn{5}{|l|}{ Wheeze at 2 years of age } \\
\hline Current wheeze & $2261 / 9953$ & 23 & $533 / 2355$ & 23 \\
\hline EVWף & $752 / 1680$ & 45 & $229 / 524$ & 44 \\
\hline MTW" & $928 / 1680$ & 55 & $295 / 524$ & 56 \\
\hline \multicolumn{5}{|l|}{ Wheeze at 4 years of age } \\
\hline Current wheeze & $1780 / 9391$ & 19 & $504 / 2609$ & 19 \\
\hline EVW" & $519 / 1423$ & 36 & $158 / 498$ & 32 \\
\hline MTW" & $904 / 1423$ & 64 & $340 / 498$ & 68 \\
\hline \multicolumn{5}{|l|}{ Wheeze at 6 years of age } \\
\hline Current wheeze & $1129 / 8393$ & 13 & $330 / 2077$ & 16 \\
\hline EVW" & $236 / 779$ & 30 & $79 / 325$ & 24 \\
\hline MTW" & $543 / 779$ & 70 & $246 / 325$ & 76 \\
\hline
\end{tabular}

EVW: episodic viral wheeze; MTW: multitrigger wheeze. " : in ALSPAC the remaining children are ethnically diverse while in LRC the remaining children are of South Asian origin. ๆ: denominator represents children with current wheeze that can be classified into EVW or MTW. Excludes children with non-classifiable wheeze (table 1) and thus does not equal the number with any current wheeze. 
or who lived in crowded homes were lower in children who participated in only one to two surveys than in those who participated in all three surveys, and lower still in children excluded from the analyses (supplementary table S2). Maternal smoking during pregnancy was more common among children with MTW than among those with EVW (supplementary table S3).

\section{Prevalence of current wheeze and wheeze phenotypes at ages 2, 4 and 6 years}

Prevalence of current wheeze in ALSPAC was $23 \%$ at age 2 years, and decreased to $13 \%$ at age 6 years (table 2). In the LRC, current wheeze decreased similarly from $23 \%$ at age 2 to $16 \%$ at age 6 years. The relative frequencies of the two phenotypes were remarkably similar in both cohorts. At age $2,45 \%$ of all classifiable wheezers in ALSPAC (44\% in the LRC) were defined as EVW; this decreased to 36\% (32\%) at age 4 and $30 \%(24 \%)$ at age 6 .

\section{Associations between wheeze phenotypes and indicators of wheeze severity}

Severity of wheezing illness as defined by the five indicators (frequency of attacks, shortness of breath, sleep disturbance, interference with activities and speech limitation) was higher for MTW than for EVW (table 3). The difference between phenotypes was larger in the LRC than in ALSPAC. For example, at age 2, the odds ratio for having MTW rather than EVW, comparing children with frequent episodes of wheeze to those with less frequent episodes, was 2.7 (95\% CI 2.2-3.2) in ALSPAC and 6.5 (95\% CI 4.1-10.4) in the LRC. In the LRC, differences between the two phenotypes became more distinct (larger odds ratios) with age.

\section{Risk of later wheeze in children with episodic viral wheeze and multitrigger wheeze}

The risk of having current wheeze 2 or 4 years later was higher for MTW than for EVW in both cohorts (supplementary tables S4 and S6). In the ALSPAC cohort, the odds ratio for wheeze at age 4 was 7.8 (95\% CI 6.5-9.3) for children with EVW at age 2 years, and 12.5 (95\% CI 10.6-14.8) for those with MTW, compared to children who did not wheeze. In the LRC, the odds ratios were 3.7 (95\% CI 2.6-5.3) and 9.9 (95\% CI 7.2-13.5). Prediction of later wheeze was stronger from age 4-6 years: in ALSPAC, the odds ratios were 26.6 (95\% CI 22.2-32.1) for MTW and 11.9 (95\% CI 9.5-14.8) for EVW at baseline (supplementary table S4, crude odds ratios). When the regression models were adjusted for wheeze severity, the difference in prognosis between the two phenotypes diminished somewhat, particularly in ALSPAC (supplementary table S4, adjusted odds ratio). The odds ratios for current wheeze 4 years later (prediction from 2 to 6 years) were lower than for the 2-year prediction intervals (supplementary table S6).

TABLE 3 Association between wheeze phenotypes and symptom severity in the Avon Longitudinal Study of Parents and Children (ALSPAC) and the Leicester Respiratory Cohort (LRC) at ages 2, 4 and 6 years

\begin{tabular}{|c|c|c|c|c|c|c|}
\hline \multirow[t]{2}{*}{ Indicators of symptom severity\# } & \multicolumn{3}{|c|}{ ALSPAC } & \multicolumn{3}{|r|}{ LRC } \\
\hline & EVW & MTW & MTW versus EVW 7 & EVW & MTW & MTW versus EVW \\
\hline Wheeze at 2 years of age $n$ & 752 & 928 & & 229 & 295 & \\
\hline Frequent attacks & 39.7 & 63.6 & $2.7(2.2-3.2)$ & 11.5 & 45.9 & $6.5(4.1-10.4)$ \\
\hline Shortness of breath & 43.3 & 58.2 & $1.8(1.5-2.2)$ & 39.9 & 76.2 & $4.8(3.3-7.0)$ \\
\hline Sleep disturbance & NA & NA & NA & 40.4 & 74.0 & $4.2(2.9-6.1)$ \\
\hline Interference with activities & NA & NA & NA & 38.0 & 73.6 & $4.5(3.1-6.6)$ \\
\hline Wheeze at 4 years of age $n$ & 519 & 904 & & 158 & 340 & \\
\hline Frequent attacks & 45.3 & 74.0 & $3.4(2.7-4.3)$ & 7.6 & 40.0 & $8.1(4.3-15.1)$ \\
\hline Shortness of breath & 50.2 & 64.1 & $1.8(1.4-2.2)$ & NA & NA & NA \\
\hline Sleep disturbance & NA & NA & NA & 41.7 & 71.3 & $3.5(2.3-5.2)$ \\
\hline Interference with activities & NA & NA & NA & 37.2 & 74.8 & $5.0(3.3-7.5)$ \\
\hline Wheeze at 6 years of age $n$ & 236 & 543 & & 79 & 246 & \\
\hline Frequent attacks & 39.6 & 64.7 & $2.8(2.0-3.8)$ & 5.1 & 41.1 & $12.9(4.6-36.4)$ \\
\hline Shortness of breath & 53.0 & 61.3 & $1.4(1.0-1.9)$ & NA & NA & NA \\
\hline Sleep disturbance & 52.4 & 62.4 & $1.5(1.1-2.1)$ & 43.0 & 67.4 & $2.7(1.6-4.6)$ \\
\hline Interference with activities & NA & NA & NA & 29.1 & 78.7 & $9.0(5.1-16.0)$ \\
\hline Speech limitation & 8.1 & 13.4 & $1.8(1.0-3.0)$ & NA & NA & NA \\
\hline
\end{tabular}

Data are presented as \% or OR $(95 \% \mathrm{CI})$, unless otherwise stated. EVW: episodic viral wheeze; MTW: multitrigger wheeze; NA: not available. ${ }^{\#}$ : definitions of severity indicators are provided in supplementary

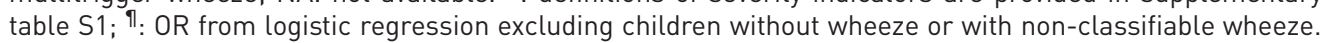


a)

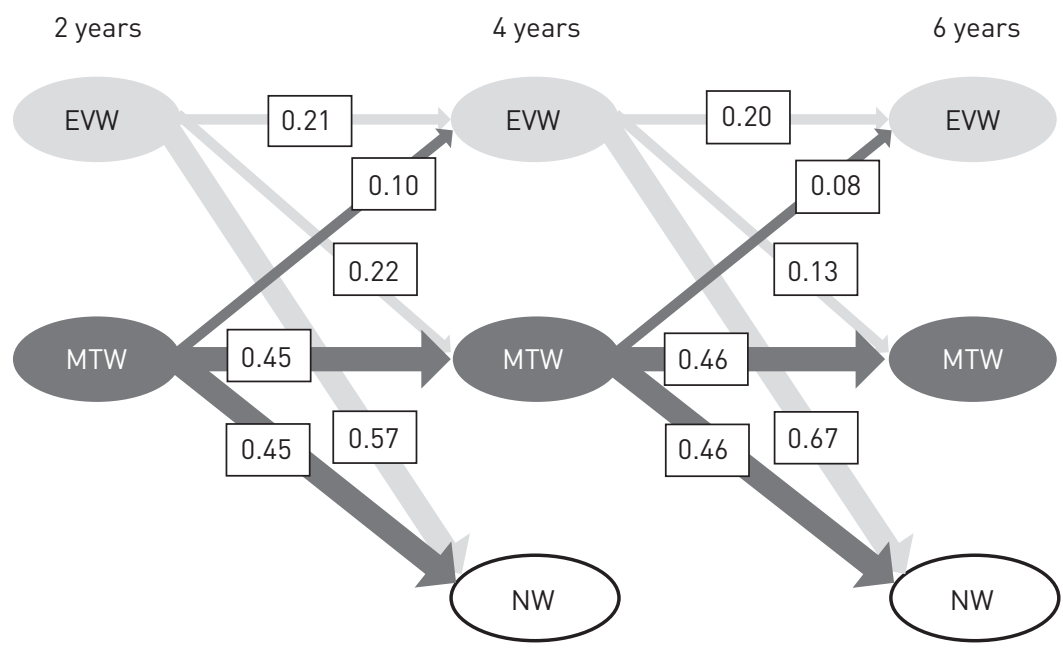

b)

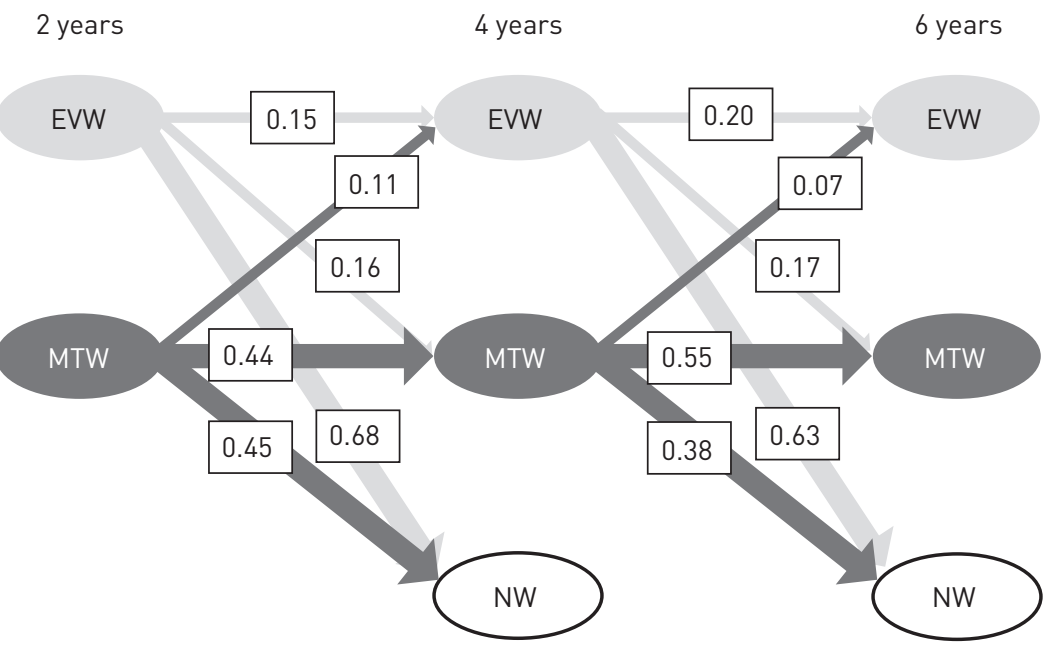

FIGURE 1 Transition probabilities from episodic viral wheeze (EVW) and multitrigger wheeze (MTW) to EVW, MTW and no wheeze (NW) for ages 2-4 years and ages 4-6 years in a) the Avon Longitudinal Study of Parents and Children and b) the Leicester Respiratory Cohort.

\section{Likelihood of keeping or switching wheeze phenotype}

The proportion of children remaining in their phenotype or transitioning to another phenotype was similar in the two cohorts (supplementary table S5 and figure 1). Among the ALSPAC children who had EVW at 2 years and who had a classifiable wheezing pattern 2 years later, $57 \%$ became asymptomatic, while $21 \%$ still had EVW and 22\% had developed MTW. Among children with MTW at age 2, 45\% became asymptomatic, $45 \%$ remained MTW and only $10 \%$ were reclassified to EVW.

Despite considerable proportions of children remitting or changing phenotype, multinomial logistic regressions showed a tendency of phenotypes to persist: RRRs were consistently higher for remaining in the same phenotype than for phenotype switching (table 4 and supplementary tables S5 and S7). Among children with EVW at age 2 years in ALSPAC, the crude RRR was 9.4 (95\% CI 7.4-11.9) for EVW (stable phenotype) but 7.7 (95\% CI 6.1-9.7) for MTW (phenotype switching) at 4 years. Among children with MTW at 2 years, the tendency for persistence was much stronger with a RRR for later MTW and EVW of 20.5 (95\% CI 16.8-24.8) and 5.9 (95\% CI 4.4-7.8), respectively. Phenotype persistence was stronger for both phenotypes from age 4 to age 6, and was strongest for MTW, with RRRs 44.9 (95\% CI 35.4-56.9) and 27.3 (95\% CI 18.9-39.6) in ALSPAC and the LRC, respectively. Although the RRRs diminished after adjustment for severity, they remained considerably higher for remaining in the same phenotype than for switching, particularly for MTW (table 4, adjusted RRR). Despite the larger proportions of children becoming asymptomatic, the RRRs for the 4-year period of age 2-6 years still revealed a tendency of phenotypes to persist (supplementary table S7). Additionally, adjusting regression models for 
TABLE 4 Likelihood of retaining the same phenotype or switching the wheeze phenotype with age in children in the Avon Longitudinal Study of Parents and Children (ALSPAC) and the Leicester Respiratory Cohort (LRC)

\begin{tabular}{|c|c|c|c|c|c|c|c|c|c|c|c|}
\hline & \multirow{2}{*}{$\begin{array}{c}\text { Age at } \\
\text { baseline years }\end{array}$} & \multirow{2}{*}{$\begin{array}{c}\text { Age at } \\
\text { follow-up years }\end{array}$} & \multirow{2}{*}{$\begin{array}{c}\text { Phenotype at } \\
\text { baseline }\end{array}$} & \multicolumn{4}{|c|}{ EVW at follow-up } & \multicolumn{4}{|c|}{ MTW at follow-up } \\
\hline & & & & $\begin{array}{c}\text { Crude RRR }{ }^{\#} \\
(95 \% \mathrm{CI})\end{array}$ & p-value ? & $\begin{array}{c}\text { Adjusted RRR } \\
\text { (95\% CI) }\end{array}$ & p-value ${ }^{\pi}$ & $\begin{array}{l}\text { Crude RRR } \\
(95 \% \mathrm{CI})\end{array}$ & p-value ๆ & $\begin{array}{l}\text { Adjusted RRR }{ }^{+} \\
(95 \% \mathrm{CI})\end{array}$ & p-value ? \\
\hline \multicolumn{12}{|l|}{ ALSPAC } \\
\hline & 2 & 4 & No wheeze & 1 & 0.004 & 1 & $<0.001$ & 1 & $<0.001$ & 1 & $<0.001$ \\
\hline & & & EVW & $9.4(7.4-11.9)$ & & $4.6(3.3-6.4)$ & & 7.7 (6.1-9.7) & & $3.2(2.3-4.3)$ & \\
\hline & & & MTW & $5.9(4.4-7.8)$ & & $2.2(1.5-3.3)$ & & $20.5(16.8-24.8)$ & & $6.2(4.6-8.4)$ & \\
\hline & 4 & 6 & No wheeze & 1 & 0.002 & 1 & $<0.001$ & 1 & $<0.001$ & 1 & $<0.001$ \\
\hline & & & EVW & $23.1(16.5-32.3)$ & & $8.0(4.9-13.1)$ & & $8.7(6.2-12.3)$ & & $2.0(1.2-3.3)$ & \\
\hline & & & MTW & $14.1(9.8-20.5)$ & & $3.3(1.9-6.0)$ & & $44.9(35.4-56.9)$ & & $6.7(4.3-10.4)$ & \\
\hline \multicolumn{12}{|l|}{ LRC } \\
\hline & 2 & 4 & No wheeze & 1 & 0.868 & 1 & 0.564 & 1 & $<0.001$ & 1 & 0.004 \\
\hline & & & EVW & $4.9(3.0-8.0)$ & & $4.1(2.2-7.5)$ & & $3.1(2.0-4.9)$ & & $1.8(1.0-3.2)$ & \\
\hline & & & MTW & $5.1(3.0-8.7)$ & & $3.3(1.4-7.7)$ & & $12.9(9.1-18.2)$ & & $4.1(2.1-7.9)$ & \\
\hline & 4 & 6 & No wheeze & 1 & 0.114 & 1 & 0.074 & 1 & $<0.001$ & 1 & $<0.001$ \\
\hline & & & EVW & $15.4(8.1-29.1)$ & & $15.5(7.3-32.9)$ & & $5.1(2.8-9.3)$ & & $4.0(2.0-8.0)$ & \\
\hline & & & MTW & $8.3(4.2-16.4)$ & & $7.0(2.6-18.9)$ & & $27.3(18.9-39.6)$ & & $15.6(8.3-29.2)$ & \\
\hline
\end{tabular}

EVW: episodic viral wheeze; MTW: multitrigger wheeze. " : relative risk ratios (RRRs) from multinomial regression analysis. To provide an example for the interpretation of the RRRs, assume that among non-wheezers at baseline the risks for EVW and no wheeze at follow-up are $4 \%$ and $90 \%$, respectively. The relative risk (RR) for EVW among non-wheezers is thus 0.044. If, in children with EVW at baseline the corresponding risks are $20 \%$ and $60 \%$, respectively, i.e. RR=0.333, this would translate to an RRR for EVW at follow-up of 7.5 (0.333/0.044). The regression analysis also included children with non-classifiable wheeze as a separate category (see table 1), but results for this category are not reported. "ी: for tests of equality of RRR between EVW and MTW at baseline. Such equality implies absence of phenotype persistence. For instance, equality of RRR for EVW at follow-up means that after excluding children with MTW at follow-up, those with EVW and MTW at baseline are equally likely to have EVW at follow-up. ${ }^{+}$: adjusted for symptom severity at baseline (frequent attacks, shortness of breath, sleep disturbance, interference with activities and speech limitation). 
sociodemographic variables and early environmental exposures only led to marginal changes in estimated RRRs (results not shown).

Statistical tests also support phenotype persistence. The p-values for equality of RRRs between EVW and MTW at baseline were all $<0.01$ except in the LRC for EVW at follow-up (table 4). These p-values remained low after adjusting for symptom severity.

\section{Discussion}

Using prospectively collected data from two independent population-based cohorts, our study found that children with MTW and EVW whose wheeze persisted over 2-year periods (ages 2-4 and 4-6 years) showed a tendency to remain in the same phenotype. This persistence was stronger for MTW than for EVW and was only partially explained by reported symptom severity. This supports the hypothesis that EVW and MTW represent distinct disease entities rather than different ends of a severity spectrum. Our study also confirms that a high proportion of early wheeze remits (approximately $60-70 \%$ of EVW and $40-45 \%$ of MTW). Despite differences in study design and methodology, results from the two cohorts were very similar.

\section{Strengths and weaknesses of the study}

Our study was based on two large, population-based cohort studies that assessed wheezing prospectively. This provided large representative samples and enabled us to use phenotype definitions that are consistent over time. Both cohorts have information on frequency and severity of wheeze, which allowed us to assess whether differences in severity explained the tendency for phenotypes to persist. Although the two cohorts use different measures of severity, the relationships between these markers and phenotypes are similar in both cohorts.

Phenotype definitions were based entirely on parental reports of symptoms during the previous 12 months. Parental assessment may be unreliable not only for the presence of wheeze, but also for wheeze severity and the presence of viral infections. In both cohorts, we defined phenotypes indirectly based on individual triggers of wheeze reported. Non-viral triggers may have been under-reported because not all possible triggers were specifically listed as response options in the questionnaires. However, in the LRC, parents' direct assessment of children's wheezing pattern shows good agreement with our phenotype definitions and does not suggest under-reporting of non-viral triggers (supplementary table S8). EVW may have been under-reported in ALSPAC, as wheeze with colds was not an explicit response option (table 1). This may explain the larger proportion of non-classifiable wheeze in ALSPAC. Although both cohorts were large and population-based, not all children participated in each survey. The samples with information available at baseline and follow-up were thus somewhat reduced and not fully representative of the entire cohorts.

\section{How do the results compare with those of other studies?}

Our study is the largest study to investigate the temporal stability of MTW and EVW and the only one to statistically test whether these phenotypes have a tendency to persist. Furthermore, it is the only study to investigate whether this phenotype persistence is explained by symptom severity - a known risk factor for the persistence of wheeze. To our knowledge, only four studies have assessed the stability of EVW and MTW over time $[22,23,26,27]$, and in these, the study populations were smaller than in either of our two cohorts. The results of these studies are summarised in supplementary table S9. Despite differences in study population and design, the proportions of children becoming asymptomatic or changing phenotype were broadly comparable to those in our study. Two of the four studies investigated both EVW and MTW, and one showed - in agreement with ours - that the proportion of children remaining in the same phenotype was larger for MTW than for EVW [22], while the other study showed greater stability for EVW [23]. However, none of these studies used regression modelling to investigate the tendency of phenotypes to persist or the extent to which such a tendency might be explained by symptom severity.

Our observation that the proportion of children with MTW increases with age while EVW decreases with age is in line with the findings of other studies $[3,6,11,28,29]$. An early cross-sectional study showed a positive correlation between age and allergy and exercise as triggers of asthma, and a negative correlation between age and respiratory infections [28]. Using partly overlapping data from the LRC, we have previously shown a decrease in the proportion of infections as an exclusive trigger among children with current wheeze, from $57 \%$ at age 1 to $21 \%$ at age 9 years, while the proportion of children also reporting other triggers increased correspondingly [29].

Similarly, our findings that MTW is associated with more severe wheeze than EVW confirm findings from other studies [6,30]. Cross-sectional surveys in Aberdeen reported less frequent episodes, and less frequent night cough, shortness of breath and chest tightness in children with EVW than in those with MTW $[6,30]$. 


\section{Interpretation}

In both cohorts, we found that RRRs for EVW at follow-up were higher for children with EVW than for those with MTW at baseline, while RRRs for MTW at follow-up were higher for children with MTW at baseline. In the absence of any phenotype stability, we would have expected these RRRs to be equal. Instead, we found that children tend to remain in the same phenotype. We then explored whether this could be explained by differences in severity. If children with MTW on average had more severe disease, children classified as MTW at baseline would tend to be reclassified as MTW at follow-up. This did, in fact, explain part of the difference; however, the direction of our findings (higher RRRs for the same phenotype) remained the same after adjusting for severity. It is possible that results are still residually confounded by unmeasured severity. Although we corrected for a wide range of measures including frequency of episodes, shortness of breath, sleep and activity disturbance, these measures were based on parental reports and may be inaccurate. We also cannot exclude the possibility that the observed stability of phenotypes was partially due to the parents' tendency to give the same, possibly inaccurate, answers to the same questions on symptoms over time.

It should be noted that the stability of MTW observed in our study is not an artefact of its definition: it might, for instance, be objected that a child by definition becomes (and remains) a multitrigger wheezer from the first time they wheeze in response to a non-viral trigger. However in our study, children were assigned to phenotypes based only on triggers of wheeze in the previous 12 months. Thus, children who wheezed only with colds during this period were classified as EVW regardless of whether they had previously had MTW. This 12-month period of observation makes sense because interval symptoms may be seasonal and a classification based on shorter periods might be strongly affected by season.

Also, our study shows that EVW in preschool children should not be equated with early transient wheeze. Indeed, after adjustment, EVW had a similar predictive value for later wheeze as MTW, particularly in the ALSPAC cohort (supplementary table S4).

We suspect that our finding may be due to the fact that differences in the underlying disease processes other than severity - cause some children to wheeze only during respiratory tract infections and others to be sensitive to other triggers. This reopens the possibility that certain therapies might indeed be more effective in certain phenotypes $[9,14,16,17]$. More research is needed to understand the underlying differences between EVW and MTW. Epidemiological studies should continue to distinguish between these phenotypes and better characterise them regarding risk factors and prognosis. While translating such knowledge to clinical management will take time, our study suggests that we should not prematurely discard these phenotypes.

\section{Conclusions}

Using data from two large population-based birth cohorts, we found that MTW, and to a lesser extent EVW, shows a tendency to persist from preschool to early-school age. While many children in both phenotypes become asymptomatic, those that continue to wheeze tend to remain in the same phenotype, though some phenotype switching does occur. The tendency to remain in the same phenotype was only partially explained by wheeze severity, suggesting that there are other differences in the underlying disease processes of children with MTW and EVW.

\section{Acknowledgements}

We are extremely grateful to all the families who took part in this study, the midwives for their help in recruiting them, and the whole ALSPAC team, which includes interviewers, computer and laboratory technicians, clerical workers, research scientists, volunteers, managers, receptionists and nurses. The UK Medical Research Council and the Wellcome Trust (grant ref: 092731) and the University of Bristol provided core support for the ALSPAC study. We are also extremely grateful to all the children and their parents for participating in the Leicester Respiratory Cohort studies. Data collection was funded by the UK National Asthma Campaign, the University Hospitals of Leicester NHS Trust (R\&D), Leicestershire and Rutland Partnership Trust, Medisearch, Trent NHS Regional Health Authority, and the UK Department of Health.

\section{References}

1 Henderson J, Granell R, Sterne J. The search for new asthma phenotypes. Arch Dis Child 2009; 94: 333-336.

2 Spycher BD, Silverman M, Kuehni CE. Phenotypes of childhood asthma: are they real? Clin Exp Allergy 2010; 40: $1130-1141$.

3 Just J, Saint Pierre P, Amat F, et al. What lessons can be learned about asthma phenotypes in children from cohort studies? Pediatr Allergy Immunol 2015; 26: 300-305.

4 Silverman M. Out of the mouths of babes and sucklings: lessons from early childhood asthma. Thorax 1993; 48: $1200-1204$

5 Silverman M, Grigg J, Mc Kean M. Virus-induced wheeze in young children - a separate disease? In: Johnston S, Papadopoulos N, eds. Respiratory Infections in Allergy and Asthma. New York, Marcel Dekker, 2002; pp. $427-471$ 
6 Wassall HJ, Devenny AM, Daud Khan S, et al. A comparison of virus-associated and multi-trigger wheeze in school children. J Asthma 2005; 42: 737-744.

7 McKean MC, Leech M, Lambert PC, et al. A model of viral wheeze in nonasthmatic adults: symptoms and physiology. Eur Respir J 2001; 18: 23-32.

8 Sonnappa S, Bastardo CM, Wade A, et al. Symptom-pattern phenotype and pulmonary function in preschool wheezers. J Allergy Clin Immunol 2010; 126: 519-526.

9 Brand PL, Baraldi E, Bisgaard H, et al. Definition, assessment and treatment of wheezing disorders in preschool children: an evidence-based approach. Eur Respir J 2008; 32: 1096-1110.

10 Spycher BD, Silverman M, Brooke AM, et al. Distinguishing phenotypes of childhood wheeze and cough using latent class analysis. Eur Respir J 2008; 31: 974-981.

11 Depner M, Fuchs O, Genuneit J, et al. Clinical and epidemiologic phenotypes of childhood asthma. Am J Respir Crit Care Med 2014; 189: 129-138.

12 Phelan PD, Robertson CF, Olinsky A. The Melbourne Asthma Study: 1964-1999. J Allergy Clin Immunol 2002; 109: 189-194.

13 British Thoracic Society. Scottish Intercollegiate Guidelines Network. British guideline on the management of asthma. Edinburgh, Health Improvement Scotland, 2016.

14 Kuehni CE. Phenotype specific treatment of obstructive airways disease in infancy and childhood: new recommendations of the Swiss Paediatric Pulmonology Group. Swiss Med Wkly 2005; 135: 95-100.

15 Roth S, Barrazzone C, Barben J, et al. Empfehlungen zur Behandlung der obstruktiven Atemwegserkrankungen im Kindesalter (SGPP/PIA-CH 2009). Paediatrica 2009; 20: 44-51.

16 Bush A. Phenotype specific treatment of asthma in childhood. Paediatr Respir Rev 2004; 5: S93-S101.

17 Brand PL, Caudri D, Eber E, et al. Classification and pharmacological treatment of preschool wheezing: changes since 2008. Eur Respir J 2014; 43: 1172-1177.

18 Schultz A, Brand PL. Episodic viral wheeze and multiple trigger wheeze in preschool children: a useful distinction for clinicians? Paediatr Respir Rev 2011; 12: 160-164.

19 Garcia-Marcos L, Martinez FD. Multitrigger versus episodic wheeze in toddlers: new phenotypes or severity markers? J Allergy Clin Immunol 2010; 126: 489-490.

20 Leonardi NA, Spycher BD, Strippoli MP, et al. Validation of the Asthma Predictive Index and comparison with simpler clinical prediction rules. J Allergy Clin Immunol 2011; 127: 1466-1472.

21 Pescatore AM, Dogaru CM, Duembgen L, et al. A simple asthma prediction tool for preschool children with wheeze or cough. J Allergy Clin Immunol 2014; 133: 111-118.

22 Schultz A, Devadason SG, Savenije OE, et al. The transient value of classifying preschool wheeze into episodic viral wheeze and multiple trigger wheeze. Acta Paediatr 2010; 99: 56-60.

23 van Wonderen KE, Geskus RB, van Aalderen WM, et al. Stability and predictiveness of multiple trigger and episodic viral wheeze in preschoolers. Clin Exp Allergy 2016; 46: 837-847.

24 Boyd A, Golding J, Macleod J, et al. Cohort profile: The 'children of the 90s' - the index offspring of the Avon Longitudinal Study of Parents and Children. Int J Epidemiol 2013; 42: 111-127.

25 Kuehni CE, Brooke AM, Strippoli M-PF, et al. Cohort profile: the Leicester respiratory cohorts. Int J Epidemiol 2007; 36: 977-985.

26 Topal E, Bakirtas A, Yilmaz O, et al. Short-term follow-up of episodic wheeze and predictive factors for persistent wheeze. Allergy Asthma Proc 2013; 34: e42-e46.

27 Kappelle L, Brand PL. Severe episodic viral wheeze in preschool children: high risk of asthma at age 5-10 years. Eur J Pediatr 2012; 171: 947-954.

28 Sarafino EP, Paterson ME, Murphy EL. Age and the impacts of triggers in childhood asthma. J Asthma 1998; 35: 213-217.

29 Strippoli MP, Spycher BD, Pescatore AM, et al. Exclusive viral wheeze and allergic wheeze: evidence for discrete phenotypes. Eur Respir J 2011; 38: 472-474.

30 Tagiyeva N, McNeill G, Russell G, et al. Two main subtypes of wheezing illness? Evidence from the 2004 Aberdeen schools asthma survey. Pediatr Allergy Immunol 2008; 19: 7-12. 